\title{
Pressure dependence of the superconducting transition temperature in nominal $\mathrm{Rb} 0.5 \mathrm{Cs} 2.5 \mathrm{C}$ 60
}

\section{Citation}

Movshovich, R., J. D. Thompson, Chia-Chun Chen, and C. M. Lieber. 1994. “Pressure Dependence of the Superconducting Transition Temperature in nominalRb0.5Cs2.5C60." Physical Review B 49 (5): 3619-21. https://doi.org/10.1103/physrevb.49.3619.

\section{Permanent link}

http://nrs.harvard.edu/urn-3:HUL.InstRepos:41417428

\section{Terms of Use}

This article was downloaded from Harvard University's DASH repository, and is made available under the terms and conditions applicable to Other Posted Material, as set forth at http:// nrs.harvard.edu/urn-3:HUL.InstRepos:dash.current.terms-of-use\#LAA

\section{Share Your Story}

The Harvard community has made this article openly available. Please share how this access benefits you. Submit a story.

Accessibility 


\title{
Pressure dependence of the superconducting transition temperature in nominal $\mathbf{R b}_{0.5} \mathrm{Cs}_{2.5} \mathrm{C}_{60}$
}

\author{
R. Movshovich and J. D. Thompson \\ Los Alamos National Laboratory, Los Alamos, New Mexico 87545 \\ Chia-Chun Chen and C. M. Lieber \\ Department of Chemistry and Division of Applied Sciences, Harvard University, Cambridge, Massachusetts 02138
}

(Received 14 May 1993)

\begin{abstract}
We report measurements of the superconducting transition temperature in a compound with a nominal composition $\mathrm{Cs}_{2.5} \mathrm{Rb}_{0.5} \mathrm{C}_{60}$ under hydrostatic pressure of up to $15.5 \mathrm{kbars}$. We observe two transitions, at temperatures $T^{*}$ and $T_{c}$ in this pelletized sample. We identify the lower temperature transition $T^{*}$ with the onset of intergranular shielding currents. The upper transition temperature $T_{c}(P)$ falls on a universal $T_{c}(P)$ curve, defined from similar data obtained previously on $\mathrm{K}_{3} \mathrm{C}_{60}$ and $\mathrm{Rb}_{3} \mathrm{C}_{60}$, after we shift the pressure axis for our data by -2.6 kbars. We identify $T_{c}$ as a superconducting transition in $\mathrm{Rb}_{2} \mathrm{CsC}_{60}$ on the basis of the compressibility analysis and published values of the lattice parameters for alkali-doped $\mathrm{C}_{60}$, as well as $T_{c}(P=0)$ and $\left(d T_{c} / d P\right)_{P=0}$.
\end{abstract}

The discovery of superconductivity in the alkali-doped fullerene compounds ${ }^{1-3}$ created a separate class of hightemperature superconductors. Superconducting transitions above $30 \mathrm{~K}$ have been observed in a number of compounds. $^{4,5}$ Both experimental $^{6-8}$ and theoretical ${ }^{9,10}$ investigations arrive at the conclusion that the superconducting transitions temperature $T_{c}$, the density of states at the Fermi surface $N\left(E_{F}\right)$, and the face-centered-cubic lattice parameter $a_{0}$ have close to linear relationships among each other. Measurements of the superconducting transition temperature as a function of applied hydrostatic pressure, where we can tune continuously the lattice parameter, are then essential for understanding the nature of superconductivity in the doped $\mathrm{C}_{60}$ compounds. The route to higher transition temperatures lies in increasing the separation between the $\mathrm{C}_{60}$ molecules without destroying the original fcc structure of $\mathrm{A}_{3} \mathrm{C}_{60}$ compounds. Superconductivity has been observed in $\mathrm{Cs}_{2} \mathrm{RbC}_{60}$ at $33 \mathrm{~K},{ }^{5}$ while attempts to synthesize $\mathrm{Cs}_{3} \mathrm{C}_{60}$ have failed, presumably due to structural instability associated with the Cs atoms being too large to fit in the tetrahedral sites. Still, by increasing the Cs content further beyond $\mathrm{Cs}_{2} \mathrm{RbC}_{60}$, a compound may result in yet higher $T_{c}$. It is to this end that we synthesized the compound with a nominal composition $\mathrm{Cs}_{2.5} \mathrm{Rb}_{0.5} \mathrm{C}_{60}$.

The nominally $\mathrm{Cs}_{2.5} \mathrm{Rb}_{0.5} \mathrm{C}_{60}$ powder samples were prepared by methods described in detail elsewhere. ${ }^{7}$ The powder was then ground, pressed into pellet, and sintered at $250^{\circ} \mathrm{C}$ for $12 \mathrm{~h}$. Initial $\mathrm{x}$-ray-diffraction analysis indicated that a part of the sample forms in an fcc lattice with a lattice parameter value of $14.625 \AA$. However, dc susceptibility measurements resulted in a superconducting transition temperature of about $30.5 \mathrm{~K}$. These values indicate that the superconducting compound does not fall on a monotonic progression of $T_{c}\left(a_{0}\right)$ that exists from $\mathrm{K}_{3} \mathrm{C}_{60}$ to $\mathrm{Rb}_{2} \mathrm{CsC}_{60}$, ${ }^{4}$ if superconductivity is due to that part of the sample with $a_{0}=14.625 \AA$. The questions that then arise are (1) is the observed $T_{c}$ associated with material having $a_{0}=14.625 \AA$ and (2) if it is, will $T_{c}$ decrease monotonically as in $\mathrm{K}$ - and $\mathrm{Rb}$-doped samples, or will it increase to join the progression at $\mathrm{Rb}_{2} \mathrm{CsC}_{60}$, presumably when their lattice constants coincide?

The second motivation for undertaking pressure studies of the superconducting transition temperature in the nominal $\mathrm{Rb}_{0.5} \mathrm{Cs}_{2.5} \mathrm{C}_{60}$ compound were the results of similar studies ${ }^{6}$ performed on two different alkali-doped $\mathrm{C}_{60}: \mathrm{K}_{3} \mathrm{C}_{60}$ and $\mathrm{Rb}_{3} \mathrm{C}_{60}$. In these cases plots of the superconducting transition temperatures vs hydrostatic pressure fall on a unique curve after an appropriate relative shift along the pressure axis was made to compensate for the difference in the lattice parameters of the two materials at ambient pressure. Investigation of other superconducting doped $\mathrm{C}_{60}$ compounds and their high-pressure behavior would address the question of whether this curve is universal for other members of this class of materials, and possibly lend support to the theoretical ideas $^{9,10}$ that emanated from the previous studies.

To answer these questions we undertook a series of ac magnetic susceptibility experiments under hydrostatic pressure. We used a self-clamping $\mathrm{Be}-\mathrm{Cu}$ pressure cell ${ }^{12}$ and Fluorinert FC-75 as a hydrostatic pressure medium. This cell is capable of generating hydrostatic pressure of up to $20 \mathrm{kbar}$. The sample was placed in one of the two identical teflon cups that served as formers for the two coils of an astatic pair. Both cups were filled with Fluorinert and the pair was inserted into another Fluorinert-filled teflon cup. Care must be taken not to expose the sample to air, and so operation described above was performed in the He-atmosphere glove box. The pressure was determined from the inductively measured superconducting transition temperature of a piece of high purity lead that was pressurized with the sample. From the sharpness of the feature in ac susceptibility curves, we deduce that the pressure was very close to uniform over the sample volume. dc magnetization measurements have been performed on a pellet sample used 
in our experiment, and fractional diamagnetism was estimated to be $1-2 \%$ of $1 / 4 \pi$ at $5 \mathrm{~K}$ for an applied field of $10 \mathrm{Oe}$.

Figure 1 shows one of the raw data traces of ac susceptibility as a function of temperature at the nominal pressure of 1 bar. Most of such traces displayed not one, but two kinks in the curves, marked as $T_{c}$ and $T^{*}$ in the figure, that were separated by roughly $1.5 \mathrm{~K}$. The origin of the two kinks may be the presence of two different superconducting phases in our sample. From the magnitude of the change in slope at both transitions we would then estimate the ratio of 4 to 1 of the low to high transition temperatures phases for our sample. However, similar behavior was observed for both $K_{3} C_{60}$ and $R_{3} C_{60}$ compounds, ${ }^{6,11}$ where there was good reason to believe that only one superconducting phase was present. In those cases the lower temperature feature was attributed to the onset of intergranular coherence, with intergranular supercurrents and correspondingly higher shielding fraction. The ratios of $4: 1$ is also similar to those observed for these binary compounds. We therefore identify only $T_{c}$ as a superconducting transition temperature for our sample.

We can plot our reduced data of $T_{c}(P)$ in a manner similar to that of Sparn et al. ${ }^{6}$ where the pressure data for $\mathrm{K}$-doped samples were shifted along the pressure axis by $10.6 \mathrm{kbar}$ to bring the data for both $\mathrm{K}$ - and $\mathrm{Rb}$-doped $\mathrm{C}_{60}$ on a single curve. The resulting graph is shown in Fig. 2, where the pressure shift for $T_{c}$ of our sample is -2.6 kbars, consistent with a larger unit-cell volume relative to $\mathrm{Rb}_{3} \mathrm{C}_{60}$. The data for both transitions join the previous data for $\mathrm{K}$ - and $\mathrm{Rb}$-doped samples very well. That fact should be taken as an indication that superconductivity is not due to the phase with a lattice parameter of $a_{0}=14.625 \AA$, since it falls off the common $T_{c}\left(a_{0}\right)$ trend, and therefore we should not expect it to follow the common $T_{c}(P)$ trend as well. From these data and the published values of the lattice parameters ${ }^{4}$ for various

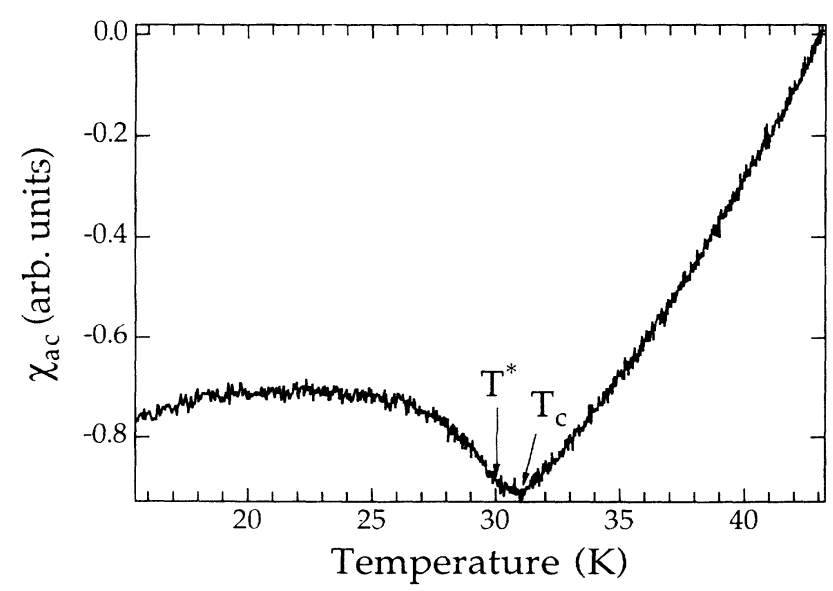

FIG. 1. ac susceptibility of nominal $\mathrm{Cs}_{2.5} \mathrm{Rb}_{0.5} \mathrm{C}_{60}$ as a function of temperature for a single temperature sweep at an applied pressure of about $1 \mathrm{kbar}$. The origins of the two transitions marked as $T^{*}$ and $T_{c}$ are discussed in the text.

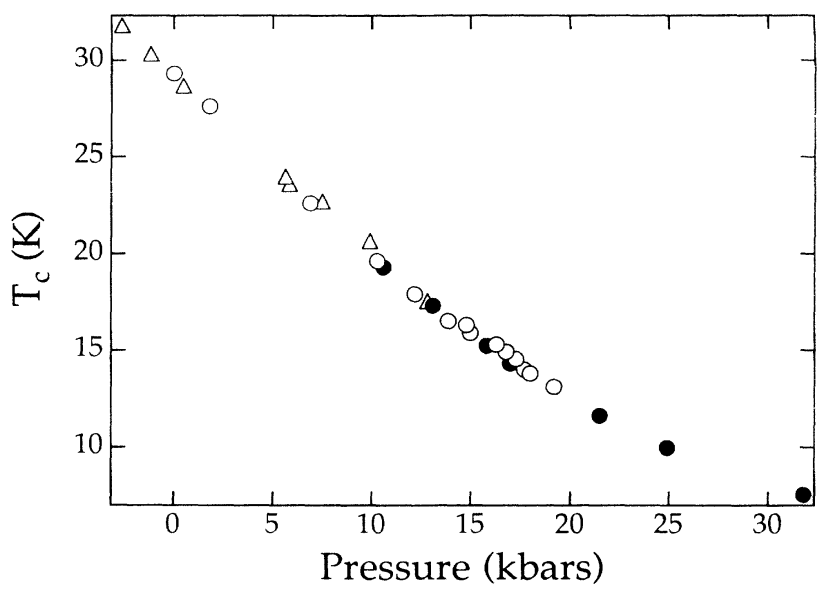

FIG. 2. Superconducting transition temperatures as a function of pressure for alkali-doped $\mathrm{C}_{60}$. $\bullet-\mathrm{K}_{3} \mathrm{C}_{60}$, data taken from Ref. 6, shifted by 10.6 kbars; $\bigcirc-\mathrm{Rb}_{3} \mathrm{C}_{60}$, data taken from Ref. 6; $\triangle-T_{c}$, shifted by -2.6 kbars.

alkali-metal dopants of $\mathrm{C}_{60}$, we can derive the linear compressibility to aid us in identifying the superconducting phase in the compound. If we take the higher temperature transition $T_{c}$ as that due to $\mathrm{Rb}_{2} \mathrm{CsC}_{60}$, we obtain the compressibility of $1.5 \times 10^{-2} \mathrm{GPa}^{-1}$ using the values of $a_{0}=14.436 \AA$ for $\mathrm{Rb}_{3} \mathrm{C}_{60}$ and $a_{0}=14.493 \AA$ for $\mathrm{Rb}_{2} \mathrm{CsC}_{60}$. Comparison with the compressibility values for $\mathrm{K}$ - and $\mathrm{Rb}$-doped samples $\left(1.2 \times 10^{-2}\right.$ and $1.5 \times 10^{-2}$ $\mathrm{GPa}^{-1}$, respectively) suggests that this identification is the correct one. The initial slope $\left(d T_{c} / d P\right)_{P=0}$ $=-0.98 \pm 0.09 \mathrm{~K} / \mathrm{kbar}$ is virtually identical to that for $\mathrm{Rb}_{3} \mathrm{C}_{60}$, ${ }^{6}$ and the superconducting transition temperature $T_{c}(P=0)=31.8 \pm 0.2 \mathrm{~K}$ is close to $31.3 \mathrm{~K}$ published previously ${ }^{4}$ for $\mathrm{Rb}_{2} \mathrm{CsC}_{60}$. On the basis of the above analysis we identify the superconducting phase in our compound as $\mathrm{Rb}_{2} \mathrm{CsC}_{60}$. We therefore believe that structural decomposition of the nominally $\mathrm{Cs}_{2.5} \mathrm{Rb}_{0.5} \mathrm{C}_{60}$ compound into $\mathrm{CsRb}_{2} \mathrm{C}_{60}$, a Cs-rich stable phase $\left(\mathrm{Cs}_{4} \mathrm{C}_{60}\right.$ and/or $\mathrm{Cs}_{6} \mathrm{C}_{60}$ ) and pure $\mathrm{C}_{60}$ is responsible for our observations.

In conclusion, we have investigated the superconducting transition in a compound of nominal composition $\mathrm{Cs}_{2.5} \mathrm{Rb}_{0.5} \mathrm{C}_{60}$ under hydrostatic pressure of up to 15.5 kbars. We see two features in the ac susceptibility versus temperature plots and attribute the lower temperature feature at $T^{*}$ as a signature of the onset of the intergranular coherence. On the basis of compressibility $\left(d T_{c} / d P\right)_{P=0}$, and $T_{c}(P=0)$ analyses, we identify the higher-temperature feature at $T_{c}$ with the superconducting transition in the $\mathrm{Rb}_{2} \mathrm{CsC}_{60}$ phase. We see a decrease with pressure of the superconducting transition temperature at the rate of $-0.98 \pm 0.09 \mathrm{~K} / \mathrm{kbar}$. This value is identical to that for the $\mathrm{Rb}_{3} \mathrm{C}_{60}$, perhaps indicating a more important role played by the smaller volume tetrahedral sites in regard to compressibility of the fcc phase of the alkali-doped $\mathrm{C}_{60}$. Transition temperature $T_{c}$ curves as a function of pressure can be shifted along the pressure axis to fall on the curve common with $\mathrm{Rb}$ - and 
$\mathrm{K}$-doped $\mathrm{C}_{60}$. The temperature of the superconducting transition is therefore independent of the particular alkali dopant, but rather is just a function of the inter- $\mathrm{C}_{60}$ spacing.
Work at Los Alamos was performed under the auspices of the U.S. Department of Energy, research at Harvard University was partially supported by the David and Lucile Packard Foundation.
${ }^{1}$ A. F. Hebbard et al., Nature (London) 350, 600 (1991).

${ }^{2}$ M. J. Rosseinsky et al., Phys. Rev. Lett. 66, 2830 (1991).

${ }^{3} \mathrm{~K}$. Holczer et al., Science 252, 1154 (1991).

${ }^{4}$ R. M. Fleming et al., Nature (London) 352, 787 (1991).

${ }^{5} \mathrm{~K}$. Tanigaki et al., Nature (London) 352, 222 (1991).

${ }^{6}$ G. Sparn et al., Phys. Rev. Lett. 68, 1228 (1992).

${ }^{7}$ Chia-Chun Chen, S. P. Kelty, and C. M. Lieber, Science 253,
886 (1991).

${ }^{8}$ O. Zhou et al., Science 255, 833 (1992).

${ }^{9}$ Ming-Zhu Huang, Yong-Nian $\mathrm{Xu}$, and W. Y. Ching, Phys. Rev. B 46, 6572 (1992).

${ }^{10}$ A. Oshiyama and S. Saito, Solid State Commun. 82, 41 (1992). ${ }^{11} \mathrm{G}$. Sparn et al., Science 252, 1829 (1991).

12J. D. Tompson, Rev. Sci. Instrum. 55, 231 (1984). 